\title{
Necessary and Sufficient Condition for Nonsingular Fisher Information Matrix in ARMA Models
}

\author{
A. Ian McLeod \\ Department of Statistical and Actuarial Sciences \\ University of Western Ontario \\ London, Ontario N6A 5B7 \\ Canada
}

\begin{abstract}
It is demonstrated that a necessary and sufficient condition that the Fisher information matrix of an ARMA model be nonsingular is that the model not be redundant, that is the autoregressive and moving-average polynomials do not share common roots.
\end{abstract}

Key words and phrases: ARMA model redundancy; Asymptotic covariance matrix of ARMA model. 
The $\operatorname{ARMA}(p, q)$ model, which may be written in operator notation as, $\phi(B) z_{t}=\theta(B) a_{t}$, where $\phi(B)=1-\phi_{1} B-\ldots-\phi_{p} B^{p}, \theta(B)=1-\theta_{1} B-\ldots-$ $\theta_{q} B^{q}, a_{t}$ is white noise with variance $\sigma_{a}^{2}>0$ and $B$ is the backshift operator on $t$, is said to be not redundant if and only if $\phi(B)=0$ and $\theta(B)=0$ have no common roots.

The Fisher information matrix may be written (Box and Jenkins, 1976, p.240), $I(\phi, \theta)=\sigma_{a}^{-2} \mathrm{E}\left\{A_{t} A_{t}^{\prime}\right\}$, where $A_{t}=\left(v_{t-1}, \ldots, v_{t-p}, u_{t-1}, \ldots, u_{t-q}\right)$, $\phi(B) v_{t}=-a_{t}, \theta(B) u_{t}=a_{t}$ and $A_{t}^{\prime}$ denotes the transpose of $A_{t}$.

The inverse of this Fisher information matrix divided by the length of an observed time series can be used to obtain an estimate of the covariance matrix of the estimated parameters $\phi_{1}, \ldots, \phi_{p}, \theta_{1}, \ldots, \theta_{q}$. McLeod (1984) and Klein and Mélard (1990) have given algorithms for computing the inverse of the Fisher information matrix. It is frequently assumed without proof in the time series literature that the Fisher information matrix is nonsingular provided the ARMA model is not redundant. For examples see, Brockwell and Davis (1991, Theorem 8.11.1), and Hannan (1970, Theorem 8, p.403 and pp.413-414). Poskitt and Tremayne (1981, p.977) have proved this result but their approach is more complicated than that given below.

Theorem: The information matrix $I(\phi, \theta)$ is nonsingular if and only if the model is not redundant.

The proof of this theorem uses the following algebraic lemma.

Lemma: A necessary and sufficient condition for model redundancy is 
that there exist nonzero polynomials $\alpha(B)=\prod_{j=1}^{p-1}\left(1-E_{j} B\right)$, and $\beta(B)=$ $\prod_{j=1}^{q-1}\left(1-F_{j} B\right)$, such that

$$
\alpha(B) \theta(B)=\beta(B) \phi(B)
$$

Proof of Lemma: Let $\phi(B)=\prod_{j=1}^{p}\left(1-G_{i} B\right)$, and $\theta(B)=\prod_{j=1}^{q}\left(1-H_{i} B\right)$. Then (1.1) can be written,

$$
\prod_{i=1}^{p-1}\left(1-E_{i} B\right) \prod_{j=1}^{q}\left(1-H_{j} B\right)=\prod_{i=1}^{q-1}\left(1-F_{i} B\right) \prod_{j=1}^{p}\left(1-G_{j} B\right) .
$$

If $H_{1}=G_{1}$ then (1.2) holds by setting $E_{i}=G_{i+1},(i=1, \ldots, p-1)$ and $F_{i}=H_{i+1},(i=1, \ldots, q-1)$. Conversely, if $G_{i} \neq H_{j}, \quad \forall i, j$ then $\phi(B) / \theta(B)$ is irreducible and must be distinct from $\alpha(B) / \beta(B)$ since the degree of the polynomials occurring in the numerator and denominator is $p$ and $q$ in the latter irreducible case and $p-1$ and $q-1$ in the former.

Proof of Theorem: $I(\phi, \theta)$ is singular if and only if there exist $\alpha_{0}, \ldots, \alpha_{p-1}$ and $\beta_{0}, \ldots, \beta_{q-1}$, not all zero, such that

$$
\operatorname{Var}\left(\sum_{i=0}^{p-1} \alpha_{i} v_{t-i}+\sum_{j=0}^{q-1} \beta_{j} u_{t-j}\right)=0
$$

where $\alpha_{i} \neq 0$ for some $i$ and $\beta_{j} \neq 0$ for some $j$. This follows because $I(\phi, \theta)=\sigma_{a}^{-2} \mathrm{E}\left\{A_{t} A_{t}^{\prime}\right\}$ is nonnegative definite. Eq. (1.3) is equivalent to the condition that there exists polynomials $\alpha(B)=\alpha_{0}+\alpha_{1} B+\ldots+\alpha_{p-1} B^{p-1}$ and $\beta(B)=\beta_{0}+\beta_{1} B+\ldots+\beta_{q-1} B^{q-1}$, at least one of which is nonzero, such that

$$
\alpha(B) v_{t-1}+\beta(B) u_{t-1}=0
$$


Eq. (1.4) can now be written

$$
\frac{\alpha(B)}{\phi(B)} a_{t}-\frac{\beta(B)}{\theta(B)} a_{t}=0
$$

Multiplying (1.5) by $a_{t}$ and taking expected values, we see that $\alpha(B) / \phi(B)-$ $\beta(B) / \theta(B)=0$ which by the Lemma is a necessary and sufficient condition for model redundancy. This establishes that lack of model redundancy is a necessary and sufficient condition for the Fisher information matrix, $I(\phi, \theta)$ to be nonsingular.

\section{Acknowledgments}

Improvements suggested by two anonymous referees are acknowledged.

\section{REFERENCES}

BROCKWELL, P.J. and DAVIS, R.A. (1991), Time Series: Theory and Methods, (2nd edn), New York: Springer-Verlag.

BOX, G.E.P. and JENKINS, G.M. (1976), Time Series Analysis: Forecasting and Control, (2nd edn), San Francisco: Holden-Day.

HANNAN, E.J. (1970), Multiple Time Series, New York: Wiley.

MCLEOD, A.I. (1984), Duality and other properties of multiplicative seasonal autoregressive-moving average time series models, Biometrika 71, $207-211$. 
KLEIN, A. AND MÉLARD, G. (1990), Fisher's information matrix for seasonal autoregressive-moving average models, Journal of Time Series Analysis 11, 231-237.

POSKITT, D.S. and TREMAYNE, A.R. (1981), An approach to testing linear time series models, The Annals of Statistics 9, 974-986. 